\title{
Rate-dependent adhesion of viscoelastic contacts. Part II: numerical model and hysteresis dissipation
}

\author{
G. Violano, ${ }^{1}$ A. Chateauminois, ${ }^{2}$ and L. Afferrante ${ }^{1, *}$ \\ ${ }^{1}$ Department of Mechanics, Mathematics and Management, \\ Polytechnic University of Bari, Via E. Orabona, 4, 70125, Bari, Italy \\ ${ }^{2}$ Soft Matter Science and Engineering Laboratory (SIMM), \\ PSL Research University, UPMC Univ Paris 06, \\ Sorbonne UniversitÃ_s, ESPCI Paris, CNRS, \\ 10 rue Vauquelin, 75231 Paris cedex 05, France
}

\begin{abstract}
In this paper, we propose a numerical model to describe the adhesive normal contact between a 'rigid' spherical indenter and a viscoelastic rough substrate. The model accounts for dissipative process under the assumption that viscoelastic losses are localized at the (micro)-contact lines. Numerical predictions are then compared with experimental measurements, which show a strong adhesion hysteresis mostly due to viscous energy dissipation occurring during pull-off. This hysteresis is satisfactorily described by the contact model which allows to distinguish the energy loss due to material dissipation from the adhesion hysteresis due to elastic instability.

Our analysis shows that the pull-off force required to detach the surfaces is strongly influenced by the detachment rate and the rms roughness amplitude, but it is almost unaffected by the maximum load from which unloading starts. Moreover, the increase in the boundary line separating contact and non-contact regions, observed when moving from smooth to rough contacts, negligibly affects the viscous dissipation. Such increase is much less significant than the reduction in contact area, which therefore is the main parameter governing the strong decrease in the effective surface energy.
\end{abstract} Keywords: viscoelasticity, adhesion hysteresis, surface roughness, pull-off force.

*Electronic address: guido.violano@poliba.it 


\section{INTRODUCTION}

Fuller \& Tabor [1] firstly showed that the pull-off force, i.e. the tensile load required to detach two contacting bodies, is strongly reduced when the surface roughness is increased. More recently, Persson \& Tosatti [2] found that adhesion leads to an increase in the real contact area, even when no pull-off force is detected.

Adhesive interactions are predominant at the nanometer scale for bodies and systems with a high surface to volume ratio [3]. However, adhesion is still observed at macroscopic scales when the contacting bodies are soft. Typical examples are pressure sensitive adhesives (PSA) [4], soft rubbers [5], sthrechable electronics [6], and biomimetic devices [7].

In several experiments $[8,9]$, the detachment behaviour of soft matter is found to be rate-dependent as a result of the intrinsic viscoelasticity of the material. In such conditions, the effective work of adhesion $\Delta \gamma_{\text {eff }}$ may be strongly increased compared to the quasi-static value $\Delta \gamma_{0}$.

Usually, the contact between soft media is described by the classical JKR adhesion theory [10]. However, the JKR theory applies for purely elastic media as it neglects rate-dependence.

The coexistence of adhesion, viscoelasticity and surface roughness has been experimentally investigated in numerous works $[9,11-14]$. In such works, loading-unloading experimental curves are usually fitted by exploiting the JKR theory, with the stratagem of using different values of the work of adhesion and elastic modulus for the loading and unloading phase, respectively.

In literature, there is a lack of analytical and numerical models aimed at describing the adhesive contact of viscoelastic bodies in presence of surface roughness. Haiat \& Barthel (HB) [15] proposed an approximate model for the contact of viscoelastic rough surfaces based on the Greenwood \& Williamson (GW) model [16]. From an experimental perspective, elucidation and validation of these models using microscopic randomly rough surfaces such as abraded or bead blasted surfaces is compromised by the difficulties in the measurement of the actual distribution of micro-contact areas at the micrometer scale.

In Ref. [17], we experimentally studied the detachment of a rigid indenter from soft PolyDiMethylSiloxane (PDMS) substrates with smooth and rough surface. Specifically, roughness was obtained by texturing the surface with spherical identical micro-asperities with controlled height and spatial distributions. The designed patterned surfaces allow for a 
precise determination of the real contact area from micro-contact visualization. Interestingly, we found simple scaling laws relating the contact radius $a$ and the contact line velocity $v_{\mathrm{c}}$ measured at the macro and microscale.

Moving from the observed similarity between macro and microscale contacts, we develop a numerical model able to describe both the loading and unloading phases occurring in typical JKR tests on rough samples. Specifically, the model exploits a discrete version of the Fuller \& Tabor (FT) multiasperity model [1] to simulate the loading phase. The unloading phase is instead modelled on the basis of the solution proposed by Muller [18], with the assumption that the parameters of Muller's model, which are experimentally identified at the macroscale, can be applied to the microscale as they correspond to similar contact line velocities $v_{\mathrm{c}}$.

\section{EXPERIMENTAL SET-UP}

For details about the experimental setup, the manufacturing of the PDMS samples and the experimental procedure used during indentation tests we refer the reader to Ref. [17]. Here, we simply summarize some aspects regarding the generated patterned surfaces.

Roughness on the top of PDMS samples is obtained by texturing them with identical spherical microasperities with controlled height and spatial distributions. Patterned surfaces were obtained by moulding PDMS in Poly(MethylMethAcrylate) (PMMA) micro-milled forms using ball-end mills with a radius of $100 \mu \mathrm{m}$. In order to reduce the microscale roughness induced by the milling process and thus to enhance adhesion, the spherical cavities of the PMMA molds have been exposed to a saturated $\mathrm{CHCl} 3$ vapor for 30 minutes. As a result of surface plasticization of the glassy acrylate polymer, surface tension effects were previously found to result in a smoothening of the surface of the spherical cavities of the mold [19]. As a consequence, an increase in the radius of the spherical bumps is observed up to a $10 \%$ of the nominal value.

The size of these micro-asperities allows for an optical detection of the individual microcontact areas, which in turn provides the relationship between the real contact area $A$ and the applied normal force $F$.

The patterned surfaces are generated with a squared nominal area of $10 \mathrm{~mm}^{2}$, where asperities are randomly distributed with a density of $2 \times 10^{7} \mathrm{~m}^{-2}$. Asperities are collocated with a non-overlapping constraint. 
The first pattern is a regular square network of spherical caps having all the same height of $40 \mu \mathrm{m}$; the other patterns are characterized by spherical caps with heights distributed according to Gaussian distributions with standard deviations $\sigma=5 \mu \mathrm{m}$ and $\sigma=10 \mu \mathrm{m}$, respectively.

Indentation experiments of the glass lens on the smooth part of the PDMS sample were performed at increasing imposed loads and the contact radius was measured at each load step after the achievement of adhesive equilibrium. The resulting contact radius vs. load data were fitted according to the JKR theory. The fit allows to evaluate the values of the reduced elastic modulus $\left(E^{*}=0.83 \mathrm{MPa}\right)$ and adhesion energy $\left(\Delta \gamma_{0}=0.037 \mathrm{~J} / \mathrm{m}^{2}\right)$.

The experimental tests on the rough patterns are performed in 6 different locations for each pattern in order to have 6 realizations of the surface topography.

During loading, contact tests are performed under fixed load conditions. The applied load is incremented step by step, with an incremental step equal to $4 \mathrm{mN}$. Once each load step is reached, contact is maintained for a long time (800 s). In such conditions, we are sure that adhesive equilibrium is reached as viscoelastic effects are totally dissipated [19].

Unloading tests are instead performed by fixing the driving velocity. In such case, viscous effects occur during the detachment process and JKR theory is no longer valid.

\section{NUMERICAL MODEL}

Fig. 1A shows the frictionless adhesive contact between a 'rigid' indenter and a soft substrate of PDMS material textured with spherical micro-asperities of identical radius of curvature.

As above explained, the loading process was executed experimentally under conditions of adhesive equilibrium. Viscoelasticity is hence negligible and micro-asperities were modeled as elastic spheres in the simulation. Conversely, viscous dissipation is no longer negligible during unloading, (fig. 1B). Here, the problem is treated under the assumption that viscoelastic losses are restricted to the contact line while the bulk of the micro-contact zone behaves elastically.

This hypothesis is supported by the low glass transition temperature of the used silicone $\left(T_{g} \approx-120^{\circ} \mathrm{C}\right)$. For our PDMS, the frequency for glass transition at room temperature is about $10^{8} \mathrm{~Hz}$. A very rough estimate of the exciting frequency at the level of micro-contacts 


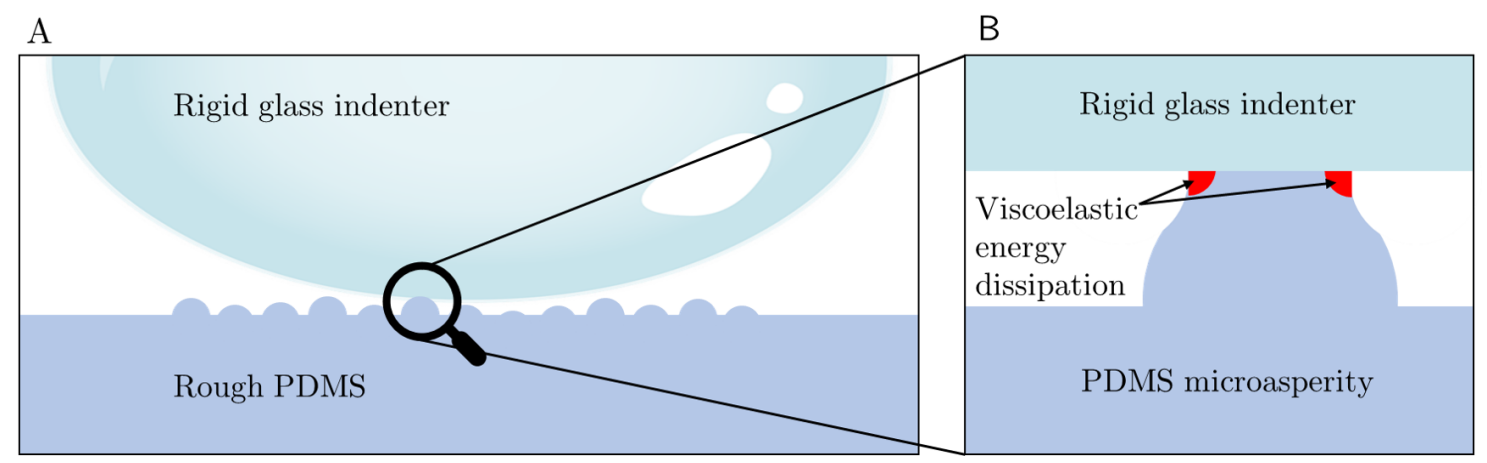

FIG. 1: A) The problem under investigations: the normal adhesive contact between a rigid smooth spherical lens and soft rough PDMS. B) Detachment of a viscoelastic micro-asperity.

is $\sim v_{\mathrm{c}} / a$, where $v_{\mathrm{c}}=-d a / d t$ is the crack tip velocity and $a$ the radius of micro-spots. As $v_{\mathrm{c}}$ is estimated $<10^{-4} \mathrm{~m} / \mathrm{s}$, assuming $a$ takes on average values of the order of $10^{-5} \mathrm{~m}$, we have exciting frequency less than $10 \mathrm{~Hz}$. Such values confirm that viscoelastic losses are negligible within the bulk of micro-asperity contacts as we are moving inside the rubbery region (or at most on the border with the transition region).

\section{A. Loading phase}

Numerical simulations of the loading phase are performed by using a discrete version of the FT multiasperity model [1], which is obtained by calculating the geometry of each asperity rather than using a statistical distribution for heights. In the original FT model the contact between two nominally flat surfaces is studied; here, we take into account the spherical shape of the indenter.

Each micro-asperity behaves as an isolated contact punch and, according to the JKR formalism [10], the contact load $F$ and approach $\delta$ are given by

$$
\begin{aligned}
F & =\frac{4}{3} \frac{E^{*} a^{3}}{R}-\sqrt{8 \pi E^{*} \Delta \gamma_{0} a^{3}} \\
\delta & =\frac{a^{2}}{R}-\sqrt{\frac{2 \pi a \Delta \gamma_{0}}{E^{*}}}
\end{aligned}
$$

where $a$ and $R$ are the contact radius and radius of curvature.

The total contact area and total applied load are obtained by summing up the contribu- 
tions of each contacting asperity. For each set of surface and contact parameters 5 numerical realizations of the surfaces were considered.

In this model, the lateral interaction between asperities is not taken into account as elastic coupling can be reasonably neglected for the considered surfaces (see Ref. [19, 20]). However, we stress that lateral interactions is instead of crucial importance when the surfaces are characterized by roughness distributed on several length scales (see, for example, [21, 22]).

\section{B. Unloading phase}

Simulations of the unloading phase are performed on the base of the solution proposed by Muller [18] and just exploited in Ref. [23]. Muller showed that the detachment process of a rigid sphere from a viscoelastic half-space can be described by a two-parameter differential equation, which in dimensionless form writes

$$
\frac{d \bar{a}}{d \bar{\delta}}=\left[\frac{\Delta \gamma_{0}}{R E^{*}}\right]^{1 / 3} \cdot \frac{1}{\beta}\left[\bar{a}^{3}\left(1-\frac{\bar{\delta}}{3 \bar{a}^{2}}\right)^{2}-\frac{4}{9}\right]^{1 / n}
$$

where $\bar{a}=a / a_{0}$ and $\bar{\delta}=\delta / \delta_{0}$, with $a_{0}=3 R\left[\pi \Delta \gamma_{0} /\left(6 E^{*} R\right)\right]^{1 / 3}$ and $\delta_{0}=$ $3 R\left[\pi \Delta \gamma_{0} /\left(6 E^{*} R\right)\right]^{2 / 3}$. The parameter $\beta$ is given by

$$
\beta=\left(\frac{6}{\pi}\right)^{1 / 3}\left(\frac{4}{9} c\right)^{1 / n} V
$$

being $c$ and $n$ characteristic constants of the material.

This model bases on two main assumptions: i) viscous effects are localized at the edge of the contact line; ii) detachment occurs under constant pull-off rate conditions.

In eq. (3), the parameter $\beta$ is proportional to the pull-off rate $V=-d \delta / d t$, while $n$ may be determined experimentally. In general, $n$ ranges from 0.1 to 0.8 [18]. The initial value $\bar{a}(\bar{\delta})$ to solve eq. (3) is returned by the classical JKR equations, which can be rewritten in dimensionless form as

$$
\begin{aligned}
& \bar{a}=\left\{\frac{1}{2}\left[1+(1+\bar{F})^{1 / 2}\right]\right\}^{2 / 3} \\
& \bar{\delta}=\left(\bar{a}^{2}+\frac{\bar{F}}{2 \bar{a}}\right)
\end{aligned}
$$


where $\bar{F}=F / F_{0}$ and $F_{0}=1.5 \pi R \Delta \gamma_{0}$. The dimensionless contact load is then calculated by

$$
\bar{F}=2 \bar{a}\left(\bar{\delta}-\bar{a}^{2}\right)
$$

The FT discrete model returns the value $F_{i}$ of each contacting asperity, being $F_{i}$ the load reached at the end of the loading process; eqs. (5) and (6) can hence be used to calculate the values of the contact radius $a_{i}$ and penetration $\delta_{i}$ at the beginning of the unloading phase.

In the experiments, unloading is performed by reducing the mean penetration $\Delta$ of the indenter in the rough surface at controlled displacement rate. A micro-asperity is assumed to jump out of contact when a critical jump-off distance is reached. Contrary to the JKR theory, jump out of contact occurs always at zero contact area.

\section{RESULTS AND DISCUSSION}

In order to apply Muller's model for each detaching micro-asperity, we have to calculate the parameters $n$ and $c$ of eqs. (3-4). As shown in Ref. [17], both parameters are scale independent. Their value can be hence obtained from contact tests on smooth PDMS by fitting the experimental data with the classical equation of Gent \& Schultz [24], relating the energy release rate $G$ to the viscoelastic losses at the crack tip

$$
G=\Delta \gamma_{0}\left[1+c \cdot v_{\mathrm{c}}{ }^{n}\right]
$$

In particular, as shown in Fig. 6 of Ref. [17], the best fit is obtained using $c=31$ and $n=0.25$. Notice that, the dimensionality of $c$ is the inverse velocity unit in power of $n$. During unloading, experiments have been performed at three different driving velocities $V$ $(V=0.02,0.002,0.0002 \mathrm{~mm} / \mathrm{s})$. Due to the compliance of the cantilever beam, the actual velocity $V_{\text {act }}$ of the indenter is lower than the imposed value [17]. The values of the actual velocity $V_{\text {act }}$ and the parameter $\beta$ used in Muller's model are $V_{\text {act }}=0.8 \mathrm{~V}$ and $\beta \sim 679,67.9$, $6.79 \mathrm{~mm} / \mathrm{s}$.

Figs. 2A-C show the true contact area $A$ as a function of the applied force $F$ for three values of the detachment rate $(V=0.2,2,20 \mu \mathrm{m} / \mathrm{s})$. Experimental tests are performed on rough patterns with asperities heights normally distributed with standard deviation $\sigma=5$ $\mu \mathrm{m}$. 
Open and closed symbols refer to loading and unloading experimental data, respectively. Vertical and horizontal error bars show scattering of results obtained on 6 different experiments, corresponding to 6 contact realizations. Numerical predictions of the loading phase are plotted with black dashed line, while solid lines are used for the unloading phase.

Profilometry measurements showed that the vapor treatment used to smoothening the PMMA mold did not induce any change in the standard deviation of the asperity height distribution. However, it resulted in a roughly $10 \%$ increase in the radius of curvature of some asperities. For this reason, contact simulations have been carried out on 5 numerically generated surfaces with random distributions of asperities radius of curvature. Specifically, the radius of curvature has been assumed ranging from $100 \mu \mathrm{m}$ to $110 \mu \mathrm{m}$, with an average value of $105 \mu \mathrm{m}$. The dispersion of numerical results is shown by the error bars on the solid lines.

A good agreement is found between experimental data and numerical predictions. The pull-off process is strongly influenced by the detachment rate $V$ and, as expected, the pull-off force is enhanced by increasing $V$.

Figs. 3A-C show the effect of the maximum applied preload $F_{\max }$ on the contact area vs. applied load relation. Experimental tests are performed on rough patterns with $\sigma=5$ $\mu \mathrm{m}$, for a fixed unloading detachment rate $V=2 \mu \mathrm{m} / \mathrm{s}$ and for $F_{\max }=0.012,0.025,0.035$ $\mathrm{N}$. Once again we observe a quite good agreement with numerical predictions.

Fig. 3D shows the variation of the load $F$ with the rigid displacement $\Delta$ as predicted by the numerical model. As shown in the inset, the pull-off force is found to be almost independent of the point at which unloading starts. Increasing $F_{\max }$ of a factor $\sim 3$ leads to a $74 \%$ increase in the energy loss for adhesion hysteresis. In the contact of smooth elastic bodies, JKR theory predicts the pull-off force to be independent on the magnification of the preload $F_{\max }$. This result is still valid for viscoelastic media. In the case of rough contacts, it is not completely understood how surface roughness affects the dependence of the pulloff force on the maximum applied load. Recent experimental investigations [13] show an increase in the pull-off force with $F_{\max }$. However, such findings disagree with tests on rough PDMS performed by Kesari et al. [25], which found a little, almost negligible, enhancement of the pull-off force with $F_{\max }$ in agreement with the Greenwood's statement: "...in a number of calculations the pull-off force has proved to be almost, or completely, independent of the point at which unloading starts, although the initial parts of the curve certainly do differ." 

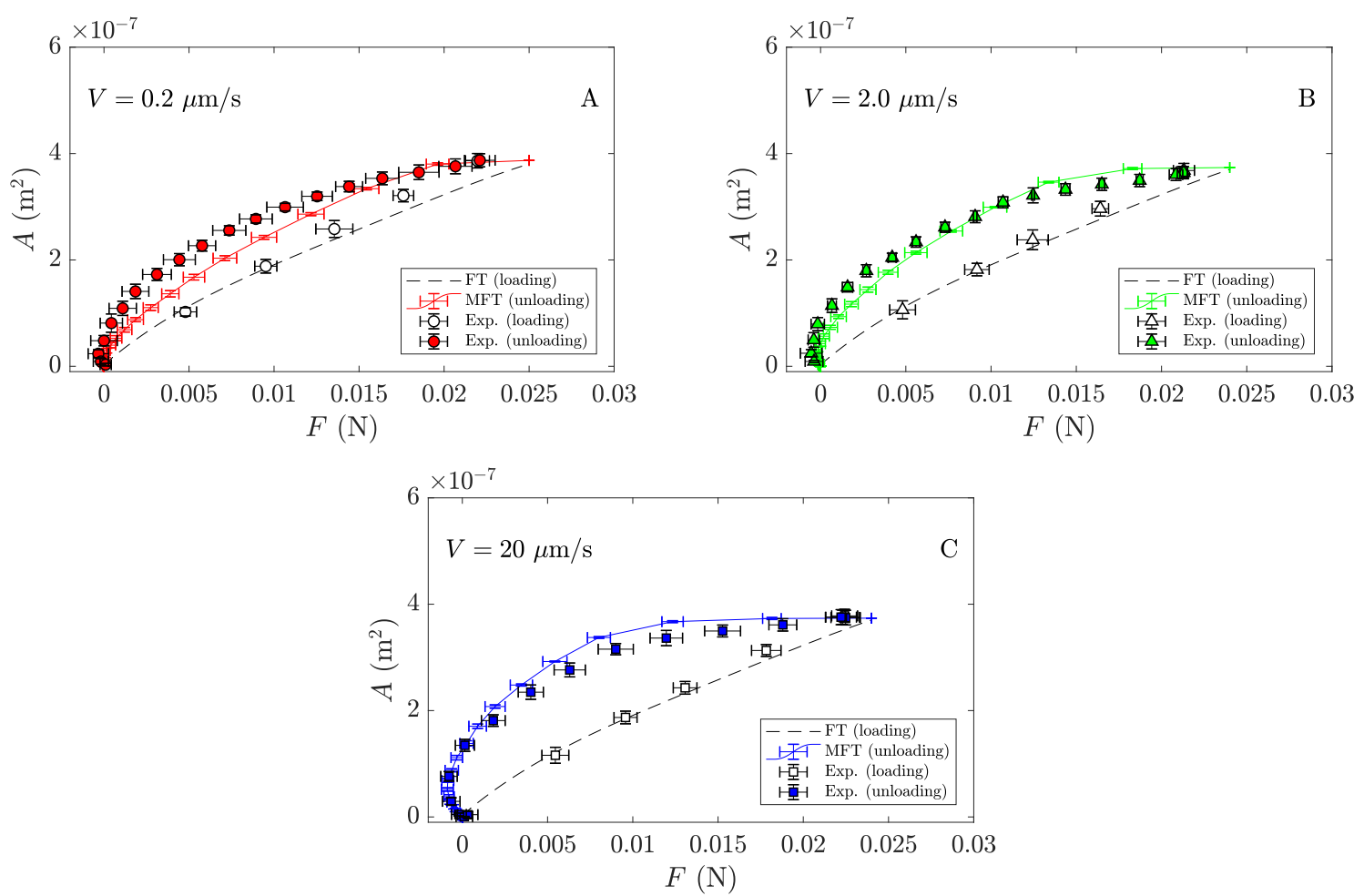

FIG. 2: The real contact area $A$ as a function of the applied load $F$. Results are obtained on rough patterns with $\sigma=5 \mu \mathrm{m}$ and for (A) $V=0.2 \mu \mathrm{m} / \mathrm{s},(B) V=2 \mu \mathrm{m} / \mathrm{s}$, and (C) $V=20 \mu \mathrm{m} / \mathrm{s}$. In all cases unloading starts after that the load $F_{\max }=0.025 \mathrm{~N}$ is reached. Experimental data are denoted with markers (open and closed symbols refer to loading and unloading, respectively). Error bars denote the standard deviation on 6 different realizations. Lines refer to numerical predictions obtained by FT model (loading) and

MFT model (unloading).

(Ref. [26]).

Figs. 4A-C show the area-load curves during loading and unloading phases for three values of $\sigma(\sigma=0,5,10 \mu \mathrm{m})$. Unloading tests were performed at fixed detachment rate $V=0.2 \mu \mathrm{m} / \mathrm{s}$. The agreement between experiments and numerical data is generally very good. Figs. 4D shows how the $F-\Delta$ relation modifies with the roughness amplitude. We notice that the pull-off force vanishes when increasing $\sigma$, in agreement with the findings of Fuller \& Tabor [1] and more recent studies [19].

However, recent experimental works find that roughness may increase adhesion. Indeed, in contact tests between a spherical tip and PMDS with nanometer scale roughness, Kesari et al. [25] found an 'optimal roughness' value, which maximizes the hysteretic energy loss.

Moreover, Dalvi et al. [27] performed adhesion measurements on soft elastic PDMS hemispheres in contact with polycrystalline diamond rough substrates. Loading-unloading tests 

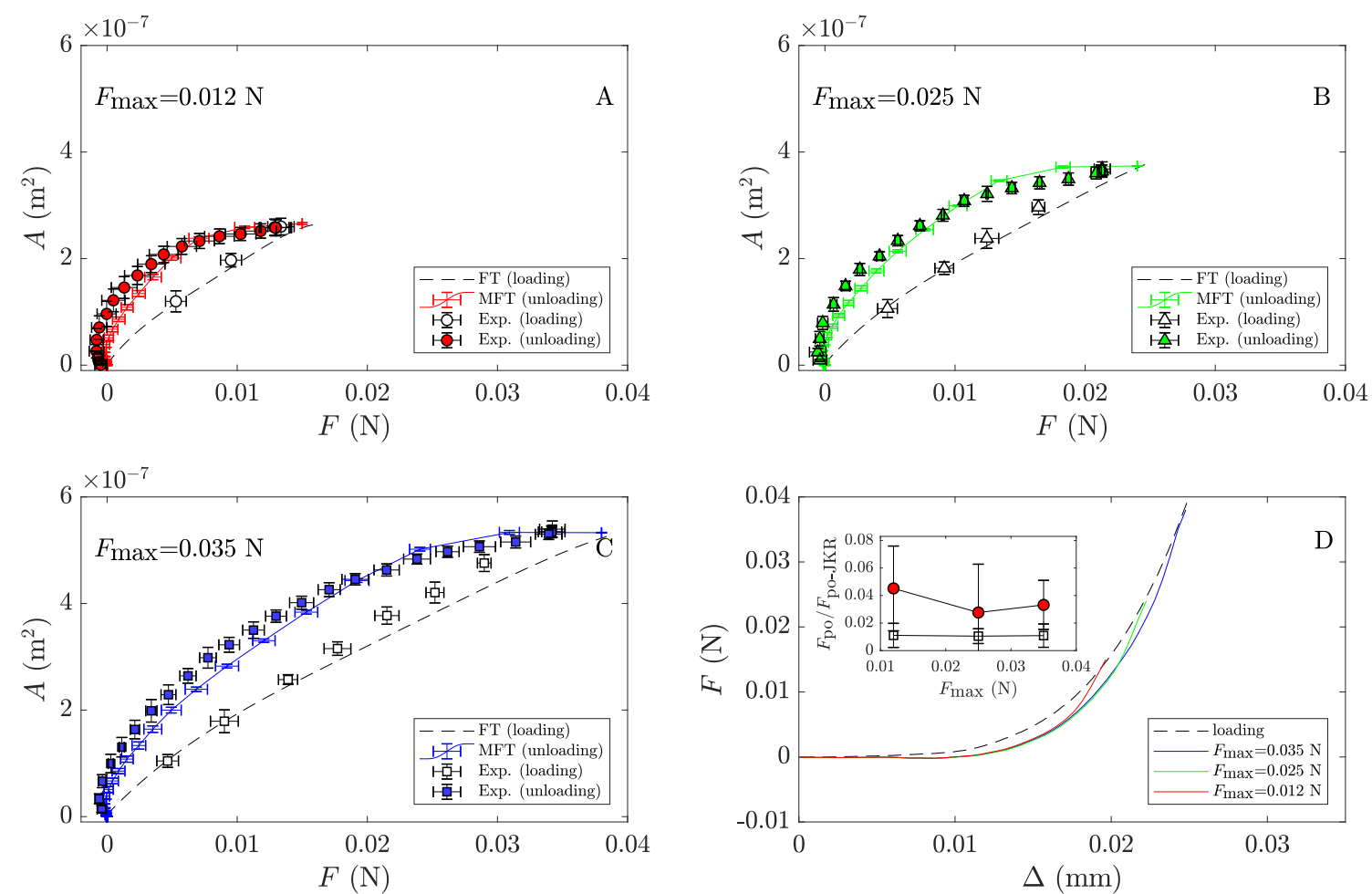

FIG. 3: The real contact area $A$ as a function of the applied load $F$. Results are obtained on rough patterns with $\sigma=5 \mu \mathrm{m}$ and for $V=2.0 \mu \mathrm{m} / \mathrm{s}$. Moreover, different values of the maximum applied preload are considered: (A) $F_{\max }=0.012 \mathrm{~N},(\mathrm{~B}) F_{\max }=0.025 \mathrm{~N}$, and

(C) $F_{\max }=0.012 \mathrm{~N}$. Experimental data are denoted with markers (open and closed symbols refer to loading and unloading, respectively). Error bars denote the standard deviation on 6 different realizations. Lines refer to numerical predictions, obtained by FT model (loading) and MFT model (unloading). D) The load $F$ as a function of the rigid

displacement $\Delta$ of the indenter. Lines refer to numerical predictions. In the inset, the normalized pull-off force $F_{\mathrm{po}} / F_{\mathrm{po}-\mathrm{JKR}}$ as a function of $F_{\text {max }}$; red circles and empty squares denote experimental and numerical data, respectively.

were conducted under quasi-static conditions, i.e. at very small values of the driving velocity of the indenter. The authors found that, even with negligible viscous effects, adhesion hysteresis still occurs because of a roughness-induced increase in contact area, in agreement with the predictions of the model of Persson \& Tosatti [2]. Moreover, Greenwood [26] extended the FT model to the unloading phase and found adhesion hysteresis occurs also in absence of viscous effects because of elastic instabilities. 

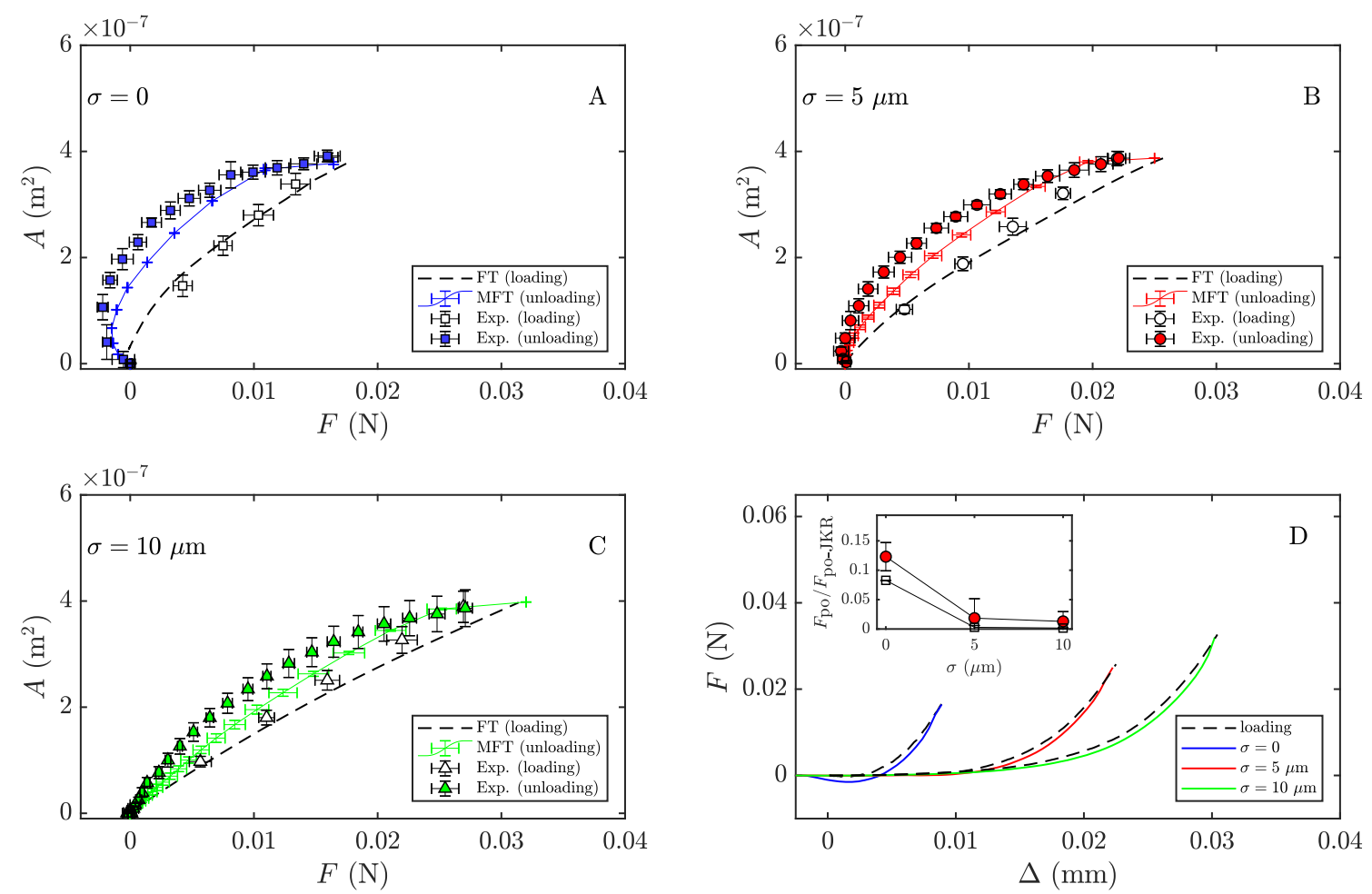

FIG. 4: The real contact area $A$ as a function of the applied load $F$. Results are obtained on rough patterns with $\sigma=0$ (A), $\sigma=5 \mu \mathrm{m}(\mathrm{B}), \sigma=10 \mu \mathrm{m}(\mathrm{C})$, and for $V=0.2 \mu \mathrm{m} / \mathrm{s}$. Experimental data are denoted with markers (open and closed symbols refer to loading and unloading, respectively). Error bars denote the standard deviation on 6 different contact realizations. Lines refer to numerical predictions, obtained by the FT (loading) and MFT (unloading) models. D) The load $F$ as a function of the rigid displacement $\Delta$ of the indenter. Lines refer to numerical predictions. In the inset, the normalized pull-off force $F_{\mathrm{po}} / F_{\mathrm{po}-\mathrm{JKR}}$ as a function of $\sigma$; red circles and empty squares denote experimental and numerical data, respectively.

\section{A. Hysteretic dissipation}

For a rigid smooth sphere approaching a flat compliant substrate, the loading-unloading path predicted by JKR theory and Muller model are shown in Fig. 5A. In JKR theory (black line), loading and unloading curves overlap and hysteretic energy loss (yellow area) is related to the elastic instabilities due to the different penetrations at which jump to contact $\left(\delta_{I N}\right)$ and detachment $\left(\delta_{O F F}\right)$ occur. In practical cases, such energy loss is usually negligible. On the contrary, in presence of viscous effects, the unloading path (red line) is rate-dependent and the hysteretic energy loss is much larger.

When a distribution of micro-asperities is textured on the substrate, the loadingunloading curves modify as shown in Fig. 5B. Numerical predictions are shown for $\sigma=5$ 

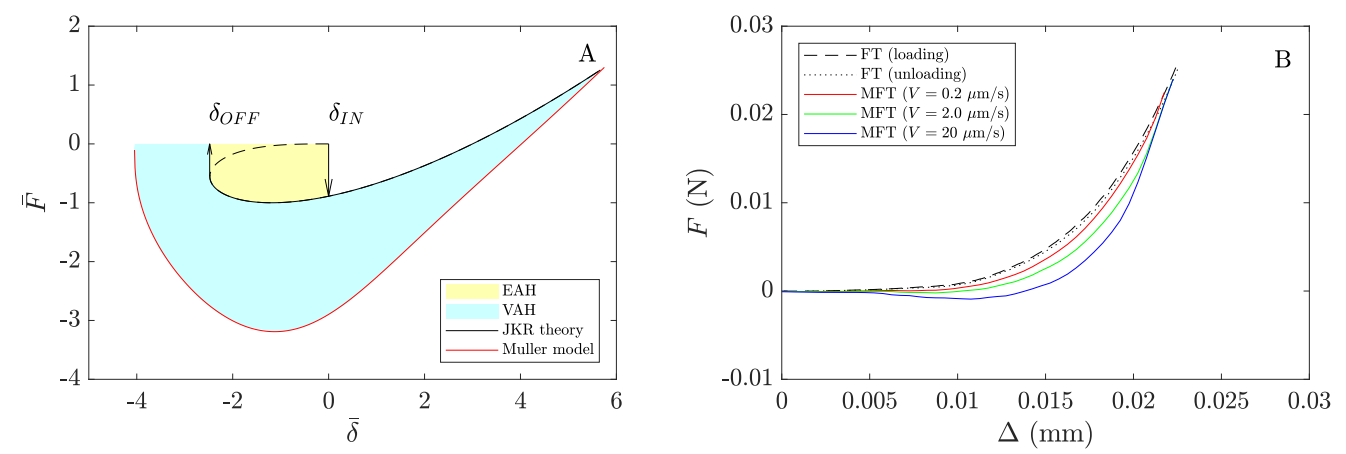

FIG. 5: A) The force-approach relation for smooth elastic spheres, as predicted by the JKR and Muller models. The yellow and cyan areas represent the energy loss due to elastic instabilities (EAH) and viscoelastic dissipation (VAH), respectively. B) The applied load $F$ as a function of the rigid displacement $\Delta$ of the indenter as predicted by the theoretical model. The loading path is plotted with black dashed lines (FT model); the unloading elastic path is plotted with black dotted line (FT model); the unloading viscoelastic path is plotted with colored solid lines (MFT model). Results are obtained on rough patterns with $\sigma=5 \mu \mathrm{m}$ and for $V=0.2,2.0,20 \mu \mathrm{m} / \mathrm{s}$ (red, green and blue).

$\mu \mathrm{m}$. Negligible hysteresis occurs when viscous effects are neglected, demonstrating that the origin of adhesion hysteresis in our experiments is strongly related to viscoelasticity. However, such result is related to have considered a single length-scale roughness. In reality, for roughness characterized by several length scales, we expect larger elastic adhesion hysteresis as a result of a more significant roughness-induced increase in contact area, as shown experimentally in Ref. [27]. Furthermore, roughness with several length scales may enhance localized phenomena of elastic instability Ref. [28].

In our experiments, surface roughness reduces the pull-off force by a factor $\sim 120$ as shown in Fig. 6A where, for $\sigma=5 \mu \mathrm{m}$, the pull-off force $F_{\text {po }}$ estimated in the tests on rough PDMS is plotted in terms of the driving velocity $V$. Notice $F_{\text {po }}$ is normalized with respect to the pull-off force $F_{\mathrm{po}-\mathrm{JKR}}=1.5 \pi R \Delta \gamma_{0}$ evaluated on a smooth sample and neglecting viscous dissipation. In Fig. 6B similar curves are given for the case of smooth sample. Results shown an increase in $F_{\text {po }}$ with $V$ and a good agreement between experimental and numerical predictions (both at macro and microscale).

Tiwari et al. [9] performing contact experiments on stiff PDMS ( $\left.E^{*} \sim 2 \mathrm{MPa}\right)$ observed that roughness leads to a decrease in $\Delta \gamma_{\text {eff }}$ of a factor $\sim 700$. However, for very soft PDMS $\left(E^{*} \sim 0.02 \mathrm{MPa}\right)$ they found an enhancement of $\Delta \gamma_{\text {eff. }}$. The latter was observed close to full-contact conditions, because of roughness-induced increase in the real contact area. In 

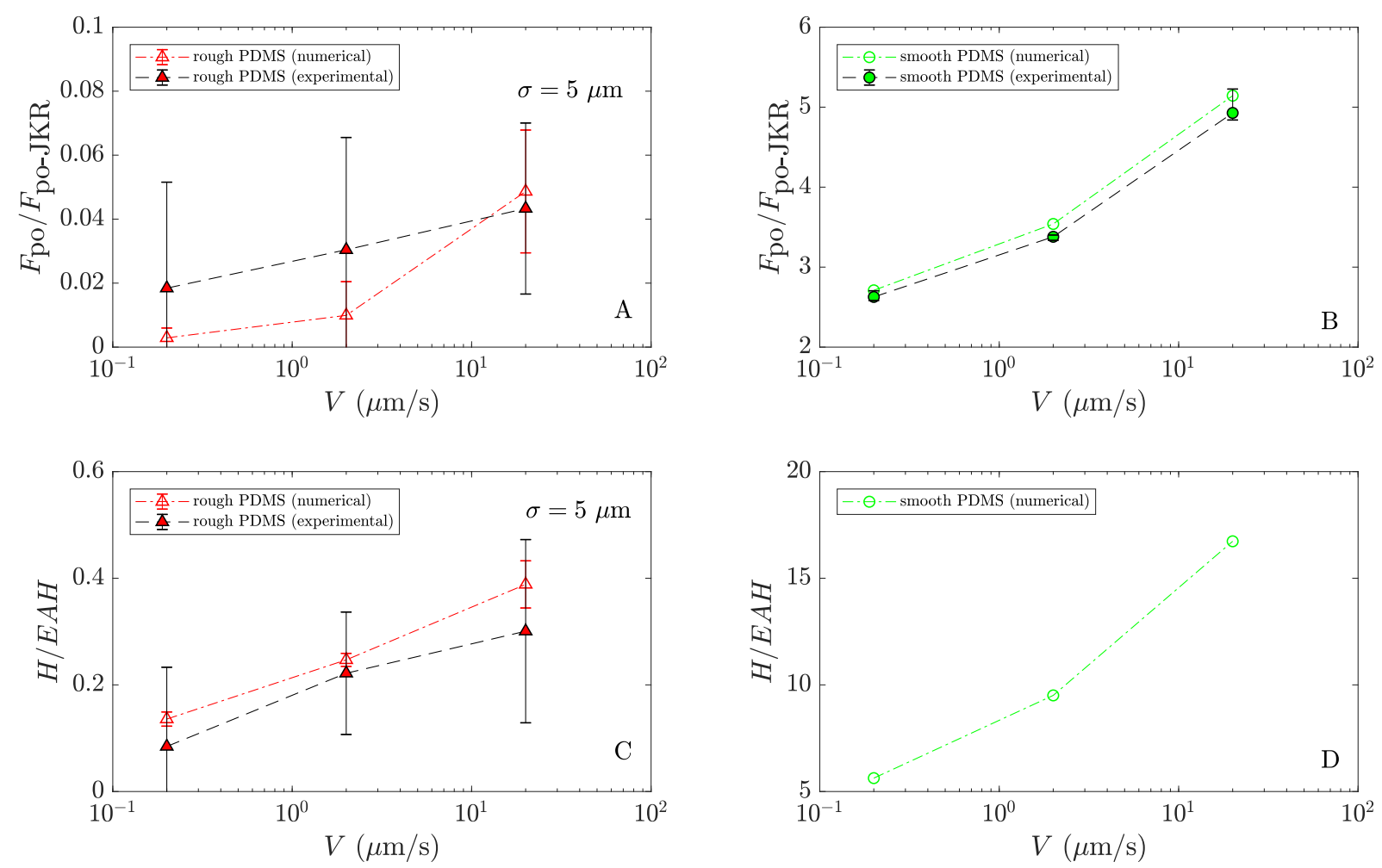

FIG. 6: A) The normalized pull-off force $F_{\mathrm{po}} / F_{\mathrm{po}-\mathrm{JKR}}$ for rough PDMS samples. Numerical (open triangles) and experimental (filled triangles) data are shown for $\sigma=5 \mu \mathrm{m}$ and $V=0.2,2.0,20 \mu \mathrm{m} / \mathrm{s}$. B) The normalized pull-off force calculated for smooth PDMS samples. C) The normalized hysteretic loss $H / E A H$ obtained on rough PDMS samples. $E A H$ is the energy dissipated as a result of the elastic instabilities predicted by the JKR theory for smooth contacts. D) The normalized hysteretic loss calculated for smooth PDMS samples.

our experiments we are far from full-contact conditions and this explains why we do not observe any increase in pull-off force moving from smooth to rough contact.

Moreover, pull-off force and effective surface energy are expected to be influenced by viscous dissipation. Under the assumption of viscous effects located near the crack tip only, the dissipation due to the crack propagation mechanism is proportional to the length $L$ of the boundary line between contact and non-contact areas [29]. Under a compressive load $F=0.02 \mathrm{~N}$, we find that the total perimeter $L_{m}$ of micro-contact spots is of the same order of magnitude of the perimeter $L_{M}$ of the smooth macro-spot $\left(L_{m} \approx 2 L_{M}\right)$. For this reason we could expect a slight increase in $\Delta \gamma_{\text {eff }}$; however, the contact area $A$ reduces more significantly (by a factor of $\sim 31$ ) as a result of the surface roughness. Such reduction is predominant and therefore governs the variation of $\Delta \gamma_{\text {eff }}$ in agreement with that observed 
in Ref. [30]: "...the effective adhesion force or surface energy per unit area can be very low - often many orders of magnitude below the value for two molecularly smooth surfaces - and is determined by a few isolated asperity contacts of low radii of curvature".

The surface roughness is expected to have the same effect on hysteretic losses in agreement with previous observations given in Szoszkiewicz et al. [31], who performing measurements of adhesion hysteresis at nanometer and micrometer length scales on mica, calcite, and metallic samples, found that hysteretic losses decrease of two orders of magnitude moving from micro to nano sized particles.

In this regard, the hysteretic loss occuring during a loading-unloading cycle is proportional to the area $H$ enclosed between the loading and unloading curves shown in Fig. 5B. Its variation with the driving velocity $V$ is plotted in Fig. $6 \mathrm{C}$, where $H$ is normalized with respect to the value calculated for a smooth sample in absence of viscous dissipation. In Fig. $6 \mathrm{D}$ the same plot is given for the case of smooth substrate. As predicted, a great reduction in adhesion hysteresis is registered when experiments (and numerical simulations) are carried out on rough samples as a result of the decrease in the real contact area. Moreover, the hysteretic losses increase with the driving velocity which is clearly related to the crack tip velocity $v_{\mathrm{c}}$ in a way that depends on the crack tip process zone.

\section{CONCLUSIONS}

In this work, we performed experimental and numerical investigations on the adhesive normal contact between a spherical indenter and viscoelastic rough substrates finding a quite good agreement.

The proposed numerical model makes use of a discrete version of the FT multiasperity model to describe the loading phase and the solution proposed by Muller [18] to characterize the unloading one. Moreover, as we can also perform the unloading process neglecting viscous effects, we can distinguish the hysteretic energy loss due to viscous dissipation from that due to the roughness-induced increase in contact area.

Both experimental and numerical results show that adhesion is strongly enhanced by increasing the detachment rate and decreasing the rms roughness amplitude, while the pulloff force is negligibly affected by the maximum applied load. This last trend is in agreement with previous studies $[25,26]$, but it seems to contradict the increase in pull-off force observed 
in Ref. [13]. However, we have to pointed out that our calculations are performed on simplified rough surfaces characterized by a single length scale.

Furthermore, we find that the increase in the effective adhesion energy with the crack tip velocity is independent on the size of the radius of curvature, depending on the viscoelastic properties of bodies exclusively. Interestingly, the increase in the perimeter of the contact line observed on rough samples negligibly affects the effective adhesion energy $\Delta \gamma_{\text {eff }}$, which is instead strongly affected by the reduction in contact area that, in our experiments, is therefore the parameter governing the change in surface energy observed when moving from smooth to rough samples.

The present study has limitations related to the simplistic description of the surface roughness, which usually presents fractal features. However, we believe it can be useful in clarifying some key points in the adhesion hysteresis of rough soft media (" Asperity approach leads naturally to an understanding of the difference between loading and unloading, and so to why there is hysteresis", Ref. [26]).

[1] Fuller, K. N. G., \& Tabor, D. (1975). The effect of surface roughness on the adhesion of elastic solids. Proceedings of the Royal Society of London. A. Mathematical and Physical Sciences, $345(1642), 327-342$.

[2] Persson, B. N. J., \& Tosatti, E. (2001). The effect of surface roughness on the adhesion of elastic solids. The Journal of Chemical Physics, 115(12), 5597-5610.

[3] Vakis, A. I., Yastrebov, V. A., Scheibert, J., Nicola, L., Dini, D., Minfray, C., ... \& Molinari, J. F. (2018). Modeling and simulation in tribology across scales: An overview. Tribology International, 125, 169-199.

[4] Benedek, I. (2004). Pressure-sensitive adhesives and applications. CRC Press.

[5] Barthel, E., \& Frétigny, C. (2009). Adhesive contact of elastomers: effective adhesion energy and creep function. Journal of Physics D: Applied Physics, 42(19), 195302.

[6] Tang, J., Li, J., Vlassak, J. J., \& Suo, Z. (2016). Adhesion between highly stretchable materials. Soft Matter, 12(4), 1093-1099.

[7] Bauer, C. T., Kroner, E., Fleck, N. A., \& Arzt, E. (2015). Hierarchical macroscopic fibrillar adhesives: in situ study of buckling and adhesion mechanisms on wavy substrates. Bioinspiration 
\& biomimetics, 10(6), 066002 .

[8] Maugis, D., \& Barquins, M. (1978). Fracture mechanics and the adherence of viscoelastic bodies. Journal of Applied Physics D: Appl. Phys. 11 1989-2023

[9] Tiwari, A., Dorogin, L., Bennett, A. I., Schulze, K. D., Sawyer, W. G., Tahir, M., ... \& Persson, B. N. J. (2017). The effect of surface roughness and viscoelasticity on rubber adhesion. Soft Matter, 13(19), 3602-3621.

[10] Johnson, K. L., Kendall, K., \& Roberts, A. D. (1971). Surface energy and the contact of elastic solids. Proceedings of the royal society of London. A. mathematical and physical sciences, 324(1558), 301-313.

[11] Lorenz, B., Krick, B. A., Mulakaluri, N., Smolyakova, M., Dieluweit, S., Sawyer, W. G., \& Persson, B. N. J. (2013). Adhesion: role of bulk viscoelasticity and surface roughness. Journal of Physics: Condensed Matter, 25(22), 225004.

[12] Martina, D., Creton, C., Damman, P., Jeusette, M., \& Lindner, A. (2012). Adhesion of soft viscoelastic adhesives on periodic rough surfaces. Soft Matter, 8(19), 5350-5357.

[13] Dorogin, L., Tiwari, A., Rotella, C., Mangiagalli, P., \& Persson, B. N. J. (2017). Role of preload in adhesion of rough surfaces. Physical review letters, 118(23), 238001.

[14] Dorogin, L., Tiwari, A., Rotella, C., Mangiagalli, P., \& Persson, B. N. (2018). Adhesion between rubber and glass in dry and lubricated condition. The Journal of chemical physics, $148(23), 234702$.

[15] Haiat, G., \& Barthel, E. (2007). An approximate model for the adhesive contact of rough viscoelastic surfaces. Langmuir, 23(23), 11643-11650.

[16] Greenwood, J. A., \& Williamson, J. P. (1966). Contact of nominally flat surfaces. Proceedings of the royal society of London. Series A. Mathematical and physical sciences, 295(1442), 300319.

[17] Violano G., Cheateaumionis A., Afferrante L. (2020). Rate-dependent adhesion of viscoelastic contacts. Part I: contact area and contact line velocity within model multi-asperity contacts with rubber.

[18] Muller, V. M. (1999). On the theory of pull-off of a viscoelastic sphere from a flat surface. Journal of Adhesion Science and Technology, 13(9), 999-1016.

[19] Acito, V., Ciavarella, M., Prevost, A. M., \& Chateauminois, A. (2019). Adhesive contact of model randomly rough rubber surfaces. Tribology Letters, 67(2), 54 . 
[20] Yashima, S., Romero, V., Wandersman, E., Frétigny, C., Chaudhury, M. K., Chateauminois, A., \& Prevost, A. M. (2015). Normal contact and friction of rubber with model randomly rough surfaces. Soft Matter, 11(5), 871-881.

[21] Violano, G., \& Afferrante, L. (2019). Contact of rough surfaces: Modeling adhesion in advanced multiasperity models. Proceedings of the Institution of Mechanical Engineers, Part J: Journal of Engineering Tribology, 1350650119838669.

[22] Violano, G., \& Afferrante, L. (2019). Modeling the adhesive contact of rough soft media with an advanced asperity model. Tribology Letters, 67(4), 119.

[23] Violano, G., \& Afferrante, L. (2019). Adhesion of compliant spheres: an experimental investigation. Procedia Structural Integrity

[24] Gent, A. N., \& Schultz, J. (1972). Effect of wetting liquids on the strength of adhesion of viscoelastic material. The Journal of Adhesion, 3(4), 281-294.

[25] Kesari, H., Doll, J. C., Pruitt, B. L., Cai, W., \& Lew, A. J. (2010). Role of surface roughness in hysteresis during adhesive elastic contact. Philosophical Magazine \& Philosophical Magazine Letters, 90(12), 891-902.

[26] Greenwood, J. A. (2017). Reflections on and extensions of the Fuller and Tabor theory of rough surface adhesion. Tribology Letters, 65(4), 159.

[27] Dalvi, S., Gujrati, A., Khanal, S. R., Pastewka, L., Dhinojwala, A., \& Jacobs, T. D. (2019). Linking energy loss in soft adhesion to surface roughness. Proceedings of the National Academy of Sciences, 116(51), 25484-25490.

[28] Carbone, G., Pierro, E., \& Recchia, G. (2015). Loading-unloading hysteresis loop of randomly rough adhesive contacts. Physical Review E, 92(6), 062404.

[29] Krick, B. A., Vail, J. R., Persson, B. N., \& Sawyer, W. G. (2012). Optical in situ micro tribometer for analysis of real contact area for contact mechanics, adhesion, and sliding experiments. Tribology letters, 45(1), 185-194.

[30] Israelachvili, J., Giasson, S., Kuhl, T., Drummond, C., Berman, A., Luengo, G., ... \& Alcantar, N. (2000). Some fundamental differences in the adhesion and friction of rough versus smooth surfaces. In Tribology Series (Vol. 38, pp. 3-12). Elsevier.

[31] Szoszkiewicz, R., Bhushan, B., Huey, B. D., Kulik, A. J., \& Gremaud, G. (2006). Adhesion hysteresis and friction at nanometer and micrometer lengths. Journal of applied physics, 99(1), 014310. 